Ophthalmologe 2012 $\cdot 109: 841-842$

DOI 10.1007/s00347-011-2506-7

(C) Springer-Verlag 2012

\section{K.-P. Steuhl}

Klinik für Erkrankungen des vorderen AugenabschnittsZentrum für Augenheilkunde, Universität Duisburg-Essen

\title{
Oberflächenrekonstruktion bei Limbusstammzellinsuffizienz
}

\author{
Aktuelle Forschung und Ausblick
}

Bei der Limbusstammzellinsuffizienz (LSI) handelt es sich um eine schwere Störung der Regeneration und Wundheilung des okulären Oberflächenepithels. Es gibt wenige hereditäre und viele erworbene Erkrankungen, die das typische Bild der LSI zeigen (s. Grüterich in diesem Heft). Infolge einer mangelhaften Regeneration des okulären Oberflächenepithels treten schwere Störungen des subjektiven Befindens bis zur funktionellen Erblindung auf.

\section{) Bei der Limbusstamm- zellinsuffizienz kommt es zu schweren Störungen des subjektiven Befindens bis zur funktionellen Erblindung}

Um das Krankheitsbild der LSI zu verstehen und eine adäquate Therapie durchführen zu können, ist ein tiefer Einblick in die Biologie des okulären Oberflächenepithels notwendig. Von entscheidender Bedeutung für das Verständnis der Regulation des Hornhautepithels ist das Verhalten der sog. Limbusstammzellen im Bereich des Übergangs des Bindehautepithels zum Hornhautepithel. Die Limbusstammzellen sind nach heutigem Kenntnisstand im basalen Epithel des Limbus in Form von Zellclustern lokalisiert. Wichtig für die Aufrechterhaltung der Stammzellcharakteristika dieser Zellen ist die sie umgebende Stammzellnische. Diese 3-dimensionale, einzigartige Mikroumgebung entscheidet mithilfe unterschiedlicher Signalgeber über das Schicksal der Stamm- zellen. Diese können sich entweder selbst erneuern oder zur Proliferation und Differenzierung angeregt werden. Die aktuelle Forschung versucht zurzeit, die exakten Mechanismen, die für die Aufrechterhaltung dieser Nische und die Kontrolle der Stammzellen verantwortlich sind, aufzuklären (s. auch Thomasen et al. in diesem Heft). Die Kenntnis der Anatomie und der Molekularbiologie dieser Vorgänge wird die Therapie der LSI entscheidend beeinflussen. Nach Aufdecken des Limbusstammzellkonzepts in den 1990er-Jahren wurde damals folgerichtig die LSI mit einer Limbusepitheltransplantation behandelt. Bei einseitiger LSI konnte sie mit gutem Erfolg autolog durchgeführt werden, bei beidseitigen Formen kam nur die Verwendung allogenen Spendergewebes infrage. Der große Nachteil der autologen Transplantation war, dass dabei oftmals Gewebe vom einzigen funktionstüchtigen Partnerauge des Patienten verwendet werden musste - bei der allogenen Transplantation war das hohe Abstoßungsrisiko gefürchtet, das eine lebenslange Immunsuppression notwendig werden ließ. Im gleichen Licht ist die Limbokeratoplastik zu sehen.

Alle Verfahren ändern zudem die Mikroumgebung der transplantierten Stammzellen, da sowohl die Blutzufuhr und die Innervation für längere Zeit (Monate) unterbrochen wird als auch die Transplantate einer veränderten Mechanik durch Lid- und Augenbewegungen ausgesetzt sind (s. auch Eberwein et al. in diesem Heft). Insbesondere um den Pool von verfügbarem autolo- gem Gewebe für die Transplantation zu vergrößern und um das Operationstrauma bei den Spendern zu reduzieren, hat vor allem die Arbeitsgruppe um Meller [1, 2] das Tissue-Engineering von limbalen Stammzellen molekularbiologisch untersucht und zur klinischen Anwendung gebracht. Nach Entnahme von $1 \times 2$ mm großen Limbusepitheltransplantaten werden ex vivo konfluierende Zellrasen gezüchtet, die mitsamt der als Matrix genutzten Amnionmembran auf den Defekt der Hornhautoberfläche aufgebracht werden. Die Kulturbedingungen werden so beeinflusst, dass sie der Mikroumgebung der Stammzellnische nahekommen. Mit dieser Methode lässt sich insbesondere mit autologem, aber auch mit allogenem Spendergewebe das korneale Oberflächenepithel stabilisieren und bei den Patienten ein signifikanter funktioneller Gewinn erreichen (s. Meller et al. in diesem Heft).

Entscheidend für die zukünftige Entwicklung wird sein, möglichst viel über die Regulation der Regeneration des okulären Oberflächenepithels zu erfahren, um die klinischen Ergebnisse bei den schweren, oft beidseitigen Limbusstammzellinsuffizienzen zu verbessern. Besonders bei den beidseitigen Formen wird in letzter Zeit auch intensiv über alternative Stammzellquellen geforscht. Aus autologem oralem Mukosaepithel kann ein Epithel mit hornhautähnlichem Phänotyp generiert werden. Die Ergebnisse hinsichtlich der Langzeitstabilität der Epithelmonolayer aus Mukosaepithel waren allerdings ernüchternd und sind diesbezüglich noch 
(2) exprivo expienten

nicht mit denen des ex vivo expandierten Limbusepithels vergleichbar [3].

Ein weiteres wichtiges Arbeitsgebiet betrifft die genetische Umprogrammierung autologer humaner Vorläuferzellen anderer Gewebe (Haarfollikel, mesenchymale Vorläuferzellen u. a.) und deren Einsatz bei der Behandlung der LSI [4]. Die möglichen Erfolge dieser Forschungsarbeiten wecken natürlich bei den Menschen, die aufgrund einer beidseitigen LSI erblindet sind, größte Hoffnungen, stecken aber leider noch in den Kinderschuhen.

Ihr

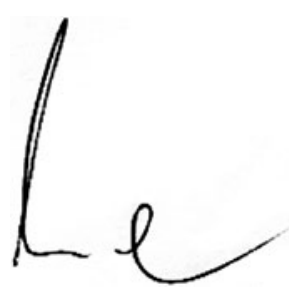

K.-P. Steuhl

\section{Korrespondenzadresse}

Prof. Dr. K.-P. Steuhl
Klinik für Erkrankungen
des vorderen
AugenabschnittsZentrum für
Augenheilkunde,
Universität Duisburg-Essen
Hufelandstr. 55, 45122 Essen
klaus-peter.steuhl@
uk-essen.de

\section{Literatur}

1. Pauklin M, Steuhl KP, Meller D (2009) Characterization of the corneal surface in limbal stem cell deficiency and after transplantation of cultivated limbal epithelium. Ophthalmology 116:1048-1056

2. Meller D, Pauklin M, Westekemper $H$, Steuhl KP (2010) Autologous transplantation of cultivated limbal epithelium. Ophthalmologe 107:11331138

3. Nakamura T, Inatomi T, Cooper LJ et al (2007) Phenotypic investigation of human eyes with transplanted autologous cultivated oral mucosal epithelial sheets for severe ocular surface diseases. Ophthalmology 114:1080-1088

4. Blazejewska EA, Schlötzer-Schrehardt U et al (2009) Corneal limbal microenvironment can induce transdifferentiation of hair follicle stem cells into corneal epithelial-like cells. Stem Cells 27:642652

\section{Leitthemenübersicht}

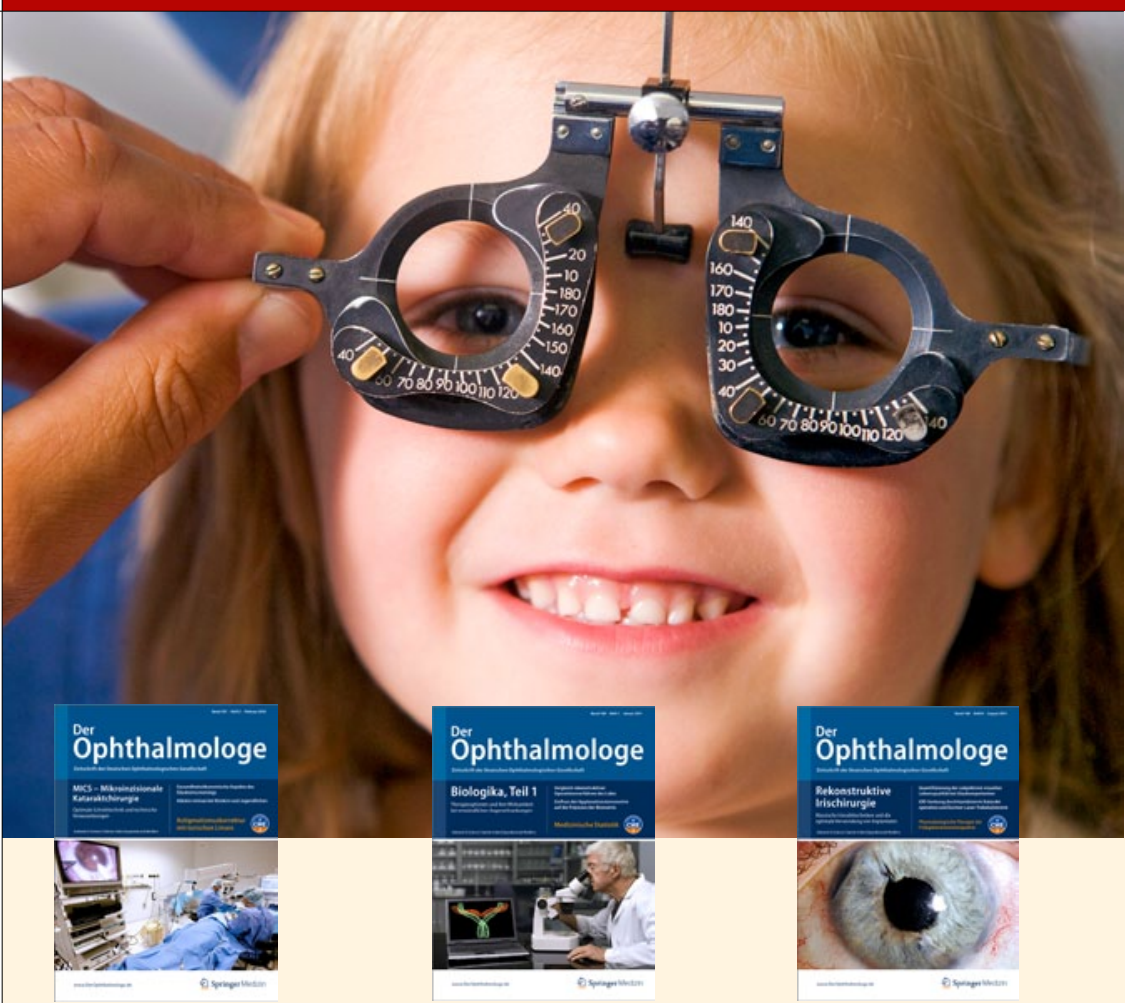

Der Ophthalmologe bietet Ihnen jeden Monat umfassende und aktuelle Beiträge zu interessanten Themenschwerpunkten aus allen Bereichen der Ophthalmologie.

Möchten Sie ein bereits erschienenes Heft nachbestellen? Einzelne Ausgaben können Sie direkt bei unserem Kundenservice zum Preis von je EUR 35,- zzgl. Versandkosten beziehen:

\section{1}

01/11 Biologika, Teil 1

02/11 Venöse retinale Gefäßverschlüsse

03/11 Biologika, Teil 2

04/11 Fallstricke der Brillenbestimmung

05/11 Orbitaerkrankungen, Teil 1

06/11 Orbitaerkrankungen, Teil 2

07/11 Glaukom im Kindesalter

08/11 Rekonstruktive Irischirurgie

09/11 Perspektiven der Hornhauttransplantation

10/11 Verätzungen und Verbrennungen des Auges

11/11 Okuläre Hypertension

12/11 Augenschmerzen aus interdisziplinärer Sicht

So erreichen Sie unseren Kundenservice:

Springer Customer Service Center GmbH

Kundenservice Zeitschriften

Haberstr. 7

69126 Heidelberg

Tel.:+49 $6221345-4303$

Fax:+49 $6221345-4229$

E-Mail:leserservice@springer.com

\section{www.DerOphthalmologe.de}

2012

01/12 Smartphones in der Augenheilkunde

02/12 Therapieansätze bei erblichen Netzhautdystrophien

03/12 Ophthalmologische Therapie in der Schwangerschaft

04/12 Funktionelle Glaukomdiagnostik

05/12 Lidchirurgie

06/12 Morbus Behçet

07/12 Submakuläre Blutung

08/12 Pseudoexfolation

09/12 Oberflächenrekonstruktion bei Limbusstammzellinsuffizienz

10/12 Myope Makuladegeneration

11/12 Frühgeborenenretinopathie

12/12 Schwindel aus interdisziplinärer Sicht 